\title{
Ansiedad dental: Evaluación y tratamiento
}

\section{Dental anxiety: Assessment and treatment}

\author{
Ríos Erazo $M^{*}$, Herrera Ronda A*, Rojas Alcayaga G*
}

\section{RESUMEN}

La ansiedad dental es un fenómeno que influye notablemente en el estado de salud oral, entorpeciendo tanto el manejo del paciente durante la atención dental como la posterior adherencia a tratamiento. Con tasas de prevalencia que van del $4 \%$ al $23 \%$, la ansiedad dental es un factor importante a considerar si se quiere mejorar la calidad de vida oral del paciente. El presente artículo propone abordar la definición de ansiedad dental, para luego identificar las causas que explican como se origina este fenómeno, además de revisar cuales son los instrumentos más utilizados para medir ansiedad dental dentro del contexto odontológico. Finalmente se revisan las intervenciones que han mostrado mayor efectividad en la reducción de este problema.

Palabras clave: Ansiedad dental, salud oral, ciencias del comportamiento.

\section{SUMMARY}

Dental anxiety is a phenomenon which significantly affects oral health status, hindering both the patient management during the dental care and the subsequent dental treatment adherence. With prevalence rates ranging from $4 \%$ to $23 \%$, dental anxiety is an important factor to consider if you want to improve the quality of the oral health of the patient. This article intends to raise the definition of dental anxiety, identify the causes behind the origin of this phenomenon and besides to check what instruments are used to measure dental anxiety in the dental context. Finally we will review the interventions that have shown greater effectiveness in reducing this problem.

Key words: Dental Anxiety, oral health, behavioral sciences.

Fecha de recepción: 12 de noviembre de 2012.

Aceptado para publicación: 18 de enero de 2013.

* Área Ciencias del Comportamiento. Departamento de Ciencias Básicas y Comunitarias. Facultad de Odontología. Universidad de Chile.

Ríos Erazo M, Herrera Ronda A, Rojas Alcayaga G. Ansiedad dental: Evaluación y tratamiento. Av. Odontoestomatol 2014; 30 (1): 39-46.

\section{ANTECEDENTES DE LA ANSIEDAD DENTAL}

La preocupación de las Ciencias Odontológicas por mejorar la salud bucal de la población es un hecho no cuestionable. La búsqueda de nuevos productos y de tecnología que aumenten la calidad de vida en relación a la salud oral de las personas avanza a pasos sorprendentes. Muchos de estos esfuerzos bus- can mejorar la adherencia al tratamiento odontológico, por lo que un abordaje integral de la enfermedad dental resulta prioritario.

Identificar las variables comportamentales que influyen en la salud dental de las personases imprescindible si se quiere dar un abordaje completo. En este sentido, la ansiedad dental es uno de los factores más 
relevantes ya que interviene enormemente, entorpeciendo tanto la adherencia a tratamiento (1-3), como la consulta de atención dental. Rowe y Moore (4) señalan que un paciente con fobia dental llega a sufrir en promedio 17,3 días de dolor antes de consultar al odontólogo. Además, la ansiedad dental es considerada un potente predictor de riesgo de caries (5).

Existe una evidencia clara de que la ansiedad dental genera una disminución del estado de salud oral del individuo (6), aumentando la presencia de caries sin tratamiento $(7,8)$ y el número de dientes cariados $(3,9)$. Además, se ha demostrado que los pacientes ansiosos tienen más probabilidades de evitar o retrasar el tratamiento y de cancelar la cita con el dentista $(3,6,10)$.

De la misma forma, distintos estudios dan cuenta de la relación indirecta existente entre la ansiedad dental y la calidad de vida en relación a salud oral (11, 12). Además, este fenómeno ha mostrado ser un muy buen predictor de problemas de manejo conducta en el sillón dental (13).

La ansiedad dental presenta una importante prevalencia, con tasas que van del $4 \%$ al $23 \%$ en países europeos $(1,10,14)$, siendo un fenómeno "sumamente extendido" en el Reino Unido (12). En Sudamérica, estudios dan cuenta de tasas del $34,7 \%$ en preescolares (15), mostrando a su vez que los padres de dichos niños también presentan una alta incidencia de ansiedad dental $(41,1 \%)(16)$. En Estados Unidos, de 10 a 12 millones de personas sufren de una severa fobia dental (el estado más grave de ansiedad dental), mientras que 35 millones de personas sufren de ansiedad dental (4).

\section{CONCEPTO DE ANSIEDAD DENTAL: CAUSAS}

En la literatura especializada muchas veces ha existido confusión entre lo que es ansiedad, miedo y fobia utilizando dichos conceptos de manera intercambiable, lo cual ha dificultado aún más la medición de la ansiedad dental.

A nivel conceptual, la ansiedad es entendida como un estado psicológico que se presenta de manera desagradable, asociado a cambios psicofisiológicos, que serían manifestación de un contenido intrapsíquico $(17,18)$. La ansiedad sería un sentimiento de miedo, donde en muchas ocasiones uno no identifica las razones de dicho sentimiento, lo que aumenta ostensiblemente la angustia manifestada. Otra definición de ansiedad dental, propuesta por AlNamankany, De Souza y Ashley (19) establece que es una respuesta multisistémica ante una creencia de peligro o amenaza, la cual es una experiencia individual, subjetiva, que varía entre las personas, y genera un serio impacto en la vida cotidiana, siendo una importante barrera para la búsqueda de atención dental.

Bajo estos antecedentes, este fenómeno debe entenderse como un concepto multidimensional, el que consta de tres componentes de respuesta que, si bien son distintos, éstos interactúan entre sí, como son el componente cognitivo, fisiológico y motor. Los aspectos cognitivos displacenteros implicarían una interferencia con la concentración, hipervigilancia atencional e incapacidad para recordar ciertos eventos. Por otro lado, los aspectos fisiológicos tienen que ver con un alto grado de activación del sistema nervioso autónomo, lo que lleva en ocasiones a una falta de respiración, transpiración y palpitaciones. Finalmente, el componente motor implica comportamientos poco ajustados y escasamente adaptativos que pueden ir desde la evitación hasta el escape de la consulta dental.

La ansiedad dental también puede presentarse de manera anticipada, con sólo pensar en el encuentro con un estimulo que provoca miedo. Es así como la persona al saber que tiene cita con el dentista, tiene la expectativa de que una experiencia aversiva se aproxima, generando altos montos de ansiedad. Otra definición de ansiedad dental es un estado de aprensión de que algo terrible pasará en relación al tratamiento dental, lo que va acompañado de un sentido de pérdida de control (20).

Por otra parte, el miedo, a diferencia de la ansiedad, no es un concepto multidimensional. Puede ser entendido como un sentimiento de inminente daño, mal, problema o una respuesta emocional. Se manifiesta como un sentimiento vago de aprehensión o inquietud, pero puede alcanzar niveles extremos como terror o pánico (6). 
La fobia, en tanto, es un grado significativo de evitación del estímulo que produce miedo, a tal punto que interfiere con la rutina normal de una persona, el funcionamiento ocupacional o académico, y las actividades y relaciones sociales (6). La fobia dental representa un tipo severo de ansiedad dental caracterizado por una marcada y persistente preocupación en relación a situaciones/objetos claramente discernibles (ej: inyecciones) o a situaciones dentales en general (20).

Las causas de porqué determinado sujeto pudiera experimentar ansiedad dental son diversas, siendo múltiples los estudios que han abordado esta temática. Locker, Shapiro y Liddell (21) demostraron que existe una importante relación entre la ansiedad dental y experiencias odontológicas negativas, estableciendo que es la naturaleza de la experiencia más que la edad en que se vivenció, el factor predictor de ansiedad dental. Dicha asociación es confirmada por el estudio de Olivera y Colares (15), quienes establecieron la asociación entre ansiedad dental y la historia de dolor dental cuando es experimentada a la edad de 5 años o menos.

Otra fuente que explica por qué se genera la ansiedad resulta ser el condicionamiento clásico, donde la sola presencia de algún estimulo que le recuerde a la persona una experiencia dental dolorosa o molesta, lo llevará a experimentar ansiedad dental (22-24).

La transmisión familiar es otra causa importante de ansiedad dental, evidenciándose una relación entre la ansiedad dental de padres y niños (25-27). Lara, Crego y Romero-Maroto (28) comprobaron la importante correlación entre ansiedad dental familiar y la de los niños, identificando el rol mediador de la ansiedad dental del padre sobre la relación entre la ansiedad de la madre y del niño, concluyendo la influencia de todos los miembros de la familia en este fenómeno.

Han surgido otras explicaciones que fundamentan porqué determinados sujetos presentan ansiedad dental, como la de Pohjola, Mattila, Joukamaa y Lahti (29), quienes concluyeron luego de evaluar a 5.241 finlandeses mayores de 30 años, que los sujetos con alexitimia (dificultad para poder identificar y expresar emociones) presentan una tendencia a sufrir ansiedad dental, lo que sugiere que algunas de estas per- sonas podrían tener una personalidad vulnerable a tener desordenes ansiosos.

\section{EVALUACIÓN DE LA ANSIEDAD DENTAL}

Existen distintos instrumentos, ampliamente utilizados en el mundo, que buscan identificar la ansiedad dental en población adulta. Uno de éstos es el Corah Dental Anxiety Scale (DAS), cuestionario de autorreporte creado por Norman Corah (30). Se compone de 4 ítems de tipo likert, que busca identificar el nivel de ansiedad del paciente cuando visitará al dentista próximamente, cuando está en la sala de espera, cuando toma los instrumentos para trabajar en sus dientes y cuando el dentista prende el micromotor para trabajar en sus dientes. Dichas preguntas tienen cinco alternativas de respuesta, obteniéndose rangos de puntaje totales que van de 4 a 20 puntos, que permiten categorizar al paciente desde las dimensiones "relajado" hasta "fobia dental". Una de las cualidades de este instrumento es que sus puntajes son altamente fidedignos, ya que como la ansiedad dental es socialmente aceptada, el tener miedo al dentista hace que poca gente tenga la necesidad de ser condescendiente cuando se le consulta al respecto.

Otro instrumento utilizado en adultos es el MDAS (Modified Dental Anxiety Scale) (31), la cual es muy similar al Corah, agregando además una pregunta referente a la inyección de la anestesia. También es un instrumento de autorreporte con cinco alternativas de respuesta que van desde la "no ansiedad" hasta "extremadamente ansioso". Los ítems son sumados para obtener el puntaje total, y mientras más puntaje, mayor es la ansiedad dental del paciente. Dicho instrumento ha mostrado una adecuada confiabilidad y validez tanto en el Reino Unido (31) como en otros países europeos (32).

En niños, los tres instrumentos más ampliamente utilizados son: el Children's Fear Survey ScheduleDental Subscale (CFSS-DS), el Venham Picture Test (VPT) y el Facial Image Scale (FIS).

El CFSS-DS, creado por Cuthbert (33) es un cuestionario que busca identificar el nivel de ansiedad dental, relacionado con diversos aspectos del tratamiento, tanto procedimientos invasivos como situa- 
ciones médicas generales. La escala esta construida para niños de entre 4 a 12 años, quienes deben responder quince ítems, las que son de tipo likert y varían de 1 a 5 ( 1 no presentan miedo y 5 manifiestan mucho miedo). Las puntuaciones obtenidas se encuentran en rangos de 15 y 75 puntos, y al obtener puntajes iguales o mayores a 45 puntos, se presentan altos montos de ansiedad. Estudios europeos, estadounidenses y chinos confirman las adecuadas propiedades psicométricas de este instrumento (34-37).

Si bien el CFSS-DS es uno de los instrumentos más utilizados, el limitado nivel de comprensión de instrucciones, y el desarrollo de la habilidad lectoescritora en niños menores de 6 años, podría dificultar la entrega de resultados veraces. Por esta razón, surgen instrumentos de respuestas más simples como el Venham Picture Test (38), donde se le presentan al niño ocho pares de figuras, las que reflejan distintas emociones y él debe escoger la que representa más fielmente su estado de ansiedad. Esta escala se puntúa de 0 (sin ansiedad) a 8 puntos (muy ansioso), teniendo como propiedades su facilidad de aplicación.

Otro instrumento utilizado para medir la ansiedad dental en niños es el Facial Image Scale (FIS), creado por Buchanan y Niven (39), el cual puede ser aplicado a niños de entre 3 a 18 años. Se compone de una hilera de cinco caras que van desde mucha felicidad a mucha infelicidad, y se le pide al niño que puntúe cual de las caras representa más como se siente en ese momento. Este instrumento muestra una adecuada facilidad de aplicación, siendo utilizado tanto en contextos europeos (40) como latinoamericanos (41).

\section{TRATAMIENTO DE LA ANSIEDAD DENTAL}

Sin duda, la ansiedad dental resulta ser un tema preocupante, que dificulta enormemente el quehacer del odontólogo, y al mismo tiempo, afecta multidimensionalmente al paciente, sobretodo en lo que respecta a la salud oral de este último. Para solucionar este fenómeno de la ansiedad dental, es que durante este último tiempo se han implementado estrategias básicas para poder disminuir estos síntomas. Sharif (42) recomienda seguir un orden lógico de manejo para el paciente con ansiedad dental. Ini- cialmente, la idea es promover la comunicación entre el dentista y el paciente, por medio de la entrega de información respecto a cuales serán los procedimientos dentales (lo que llevaría a disminuir su sensación de pérdida de control).

Existen algunos especialistas que consideran a la hipnosis como una opción válida y, si bien ésta pudiera ser una alternativa a la hora de manejar a un paciente con ansiedad dental, no existen estudios concluyentes que den cuenta de una adecuada efectividad de este tipo de técnicas, básicamente debido a la poca rigurosidad y al limitado número de estudios (43).

Bare y Dundes (44) buscaron identificar cuales eran las estrategias recomendadas por los pacientes que presentaban ansiedad dental. Un 89\% de los pacientes consideró que la música de fondo los ayudaba a relajarse, mientras que un $75 \%$ consideró que la presencia de libros y revistas disminuía su ansiedad dental.

Además de las técnicas anteriormente mencionadas, existen otras herramientas tales como el uso de técnicas de relajación y la distracción. La relajación busca una distensión tanto psíquica como muscular, facilitando la recuperación y equilibrio, hacia un estado de tranquilidad en pacientes odontológicos que presenten ansiedad, existiendo distintos estudios que dan cuenta de la efectividad de esta técnica $(45,46)$.

La distracción tiene como objetivo manejar la ansiedad, el estrés y el dolor que provocan los procedimientos clínicos, mediante la focalización de la atención fuera de los procedimientos dolorosos, siendo la música una forma de distracción. Ésta puede aliviar el dolor y la ansiedad trasladando conscientemente la atención lejos de los síntomas. Según la teoría del control del dolor, los receptores de dolor actúan en conjunto para enviar señales de sufrimiento al cerebro, por lo tanto, los distractores como la música, pueden bloquear ciertas vías de dolor y disminuir la cantidad de dolor percibido (47).

También está la percepción de control, donde se le entrega al paciente la posibilidad de detener el procedimiento dental cuando lo estime conveniente, lo que llevaría a manejar adecuadamente la ansiedad. 
A partir de estas técnicas, es que Rojas et al. buscaron evaluar la eficacia entre dos técnicas: la percepción de control y la relajación. Con respecto a los resultados, se pudo observar una disminución significativa de la ansiedad dental en los pacientes apoyados por las técnicas de percepción de control, donde un $40 \%$ de éstos se ubicaron en el rango de baja ansiedad, demostrando una eficacia mayor que la relajación (45).

Por otra parte, en niños sin alteraciones de salud, el manejo no farmacológico es la terapia de elección de la ansiedad dental. Las técnicas que han demostrado ser eficaces son el control de la voz, el manejo de la respiración, el mostrar-decir-hacer y la distracción (48). La distracción es particularmente efectiva en niños pequeños o cuando hay poco tiempo disponible para la preparación. Entre los tipos de técnicas de distracción se encuentran el cantar, ver un video, jugar un juego o cualquier otra cosa que mantenga la atención del niño fuera del procedimiento clínico (49).

Un estudio australiano demostró que las técnicas más utilizadas por los dentistas, en el manejo de la ansiedad en niños, son permitirle al menor detener el procedimiento, tener juegos en la sala de espera y la técnica mostrar-decir-hacer (50).

\section{CONCLUSIONES}

La ansiedad dental es un fenómeno que influye de sobremanera en los distintos niveles de la atención clínica, tanto en su procedimiento como en la adherencia a tratamiento, influyendo además en los indicadores de salud oral. Es por eso que resulta importante abordar este elemento desde todas sus dimensiones, identificando tanto las razones del por qué ocurre, conocer la mejor forma de evaluarlo, y establecer el mejor método de afrontamiento de este.

Un amplio rango de instrumentos ha sido desarrollado para medir ansiedad dental. Es crucial que las escalas que miden ansiedad dental sean validas y confiables ya que muchas son usadas como screening. En este sentido, si un profesional utiliza una escala mal confeccionada o sin validez, tomará decisiones en cuanto al tratamiento del paciente con información errada, lo cual puede perjudicar no sólo al paciente si no que a la relación dentista-paciente $\mathrm{y}$, finalmente, a la adherencia al tratamiento.

Por otra parte, es fundamental el conocimiento de qué es la ansiedad dental para su buen manejo posterior. Todo profesional debe abordar de manera integral al paciente, lo que necesariamente implica conocer las variables psicosociales en juego al momento de trabajar con personas, las cuales no deben ser excluidas cuando se trata de tratamientos odontológicos.

\section{AGRADECIMIENTOS}

Este trabajo fue financiado por el Fondo Nacional en Salud (FONIS), dependiente del CONICYT, a través del Proyecto FONIS SA11I2025, Ilamado: "Impacto en la adherencia a tratamiento de una intervención cognitivo-conductual para disminuir ansiedad dental en niños y niñas de 6 años beneficiarios del programa de salud oral integral en servicios de atención primaria de la Región Metropolitana".

\section{BIBLIOGRAFÍA}

1. Astrøm AN, Skaret E, Haugejorden O. Dental anxiety and dental attendance among 25-yearolds in Norway: time trends from 1997 to 2007. BMC Oral Health 2011;11:10.

2. Nicolas E, Collado V, Faulks D, Bullier B, Hennequin M. A national cross-sectional survey of dental anxiety in the French adult population. BMC Oral Health 2007; 7:12.

3. Eitner S, Wichmann M, Paulsen A, Holst S. Dental anxiety-an epidemiological study on its clinical correlation and effects on oral health. J Oral Rehabil 2006;33(8):588-93.

4. Rowe M, Moore T. Self-Report Measures of Dental Fear: Gender Differences. Am J Health Behav 1998;22(4):243-7.

5. Shapiro M, Melmed RN, Sgan-Cohen HD, Eli I, Parush S. Behavioural and physiological effect of 
dental environment sensory adaptation on children's dental anxiety. Eur J Oral Sci 2007;115 (6):479-83.

6. Armfield JM. How do we measure dental fear and what are we measuring anyway? Oral Health Prev Dent 2010;8(2):107-15.

7. Goettems ML, Ardenghi TM, Romano AR, Demarco FF, Torriani DD. Influence of maternal dental anxiety on the child's dental caries experience. Caries Res 2012;46(1):3-8.

8. Kinirons MJ, Stewart C. Factors affecting levels of untreated caries in a sample of 14-15-year-old adolescents in Northern Ireland. Community Dent Oral Epidemiol 1998;26(1):7-11.

9. Rantavuori K, Lahti S, Hausen H, Seppä L, Kärkkäinen S. Dental fear and oral health and family characteristics of Finnish children. Acta Odontol Scand 2004;62(4):207-13.

10. Armfield JM, Spencer AJ, Stewart JF. Dental fear in Australia: who's afraid of the dentist? Aust Dent J 2006;51(1):78-85.

11. Kumar S, Bhargav P, Patel A, Bhati M, Balasubramanyam G, Duraiswamy P, Kulkarni S. Does dental anxiety influence oral health-related quality of life? Observations from a cross-sectional study among adults in Udaipur district, India. J Oral Sci 2009;51(2):245-54.

12. McGrath C, Bedi R. The association between dental anxiety and oral health-related quality of life in Britain. Community Dent Oral Epidemiol 2004;32(1):67-72.

13. Gustafsson A, Broberg A, Bodin L, Berggren U, Arnrup K. Dental behaviour management problems: the role of child personal characteristics. Int J Paediatr Dent 2010;20(4):242-53.

14. Hakeberg M, Berggren U, Carlsson SG. Prevalence of dental anxiety in an adult population in a major urban area in Sweden. Community Dent Oral Epidemiol 1992;20(2):97101.
15. Oliveira MMT, Colares V. A relação entre ansiedade odontológica e a dor de dente em crianças com idade entre 18 e 59 meses: estudo em Recife, Pernambuco, Brasil. Cad. Saúde Pública 2009;25 (4):743-50.

16. Caraciolo G, Colares V. Prevalência de medo e/ ou ansiedade relacionados à visita ao dentista em crianças com 5 anos de idade nacidade do Recife. Rev Odonto Ciênc 2004;19(46):348-53.

17. Alarcón RD, Mazzotti G, Nicolini H. Psiquiatría. $2^{\mathrm{a}}$ ed. El Manual Moderno: Washington; 2005.

18. Capponi R. Psicopatología y Semiología Psiquiátrica. Editorial Universitaria: Santiago; 2002.

19. Al-Namankany A, De Souza M, Ashley P. Evidence-based dentistry: analysis of dental anxiety scales for children. Br Dent J 2012;212 (5):219-22.

20. Klingberg G, Broberg AG. Dental fear/anxiety and dental behavior management problems in children and adolescents: a review of prevalence and concomitant psychological factors. Int $\mathrm{J}$ Paediatr Dent 2007;17(6):391-406.

21. Locker D, Shapiro D, Liddell A. Negative dental experiences and their relationship to dental anxiety. Community Dent Oral Epidemiol 1996; 13(2):86-92.

22. Milgrom P, Mancl L, King B, Weinstein P. Origins of childhood dental fear. Behav Res Ther 1995;33 (3):313-9.

23. Poulton R, Waldie KE, Thomson WM, Locker D. Determinants of early-vs late-onset dental fear in a longitudinal-epidemiological study. Behav Res Ther 2001;39(7):777-85.

24. Townend E, Dimigen G. Fung D. A clinical study of child dental anxiety. Behav Res Ther 2000;38 (1):31-46.

25. Lee CY, Chang YY, Huang ST. The clinically related predictors of dental fear in Taiwanese children. Int J Paediatr Dent 2008;18(6):415-22. 
26. Nuttall NM, Gilbert A, Morris J. Children's dental anxiety in the United Kingdom in 2003. J Dent 2008;36(11):857-60.

27. Boman UW, Lundgren J, Elfström ML, Berggren U. Common use of a Fear Survey Schedule for assessment of dental fear among children and adults. Int J PaediatrDent 2008;18(1):70-6.

28. Lara A, Crego A, Romero-Maroto M. Emotional contagion of dental fear to children: the fathers' mediating role in parental transfer of fear. Int J Paediatr Dent 2012;22(5):324-30.

29. Pohjola V, Mattila AK, Joukamaa M, Lahti S. Dental fear and alexithymia among adults in Finland. Acta Odontol Scan 2011;69(4):243-7.

30. Corah NL. Development of a dental anxiety scale. J Dent Res 1969;48(4):596.

31. Humphris GM, Morrison T, Lindsay SJ. The Modified Dental Anxiety Scale: validation and United Kingdom norms. Community Dent Health 1995; 12(3):143-50.

32. Humphris GM, Freeman R, Campbell J, Tuutti H, D'Souza V. Further evidence for the reliability and validity of the Modified Dental Anxiety Scale. Int Dent J 2000;50(6):367-70.

33. Cuthbert MI, Melamed BG. A screening device: children at risk for dental fears and management problems. ASDC J Dent Child 1982;49(6):4326.

34. Alvesalo I, Murtomaa H, Milgrom P, Honkanen A, Karjalainen M, Tay KM. The Dental Fear Survey Schedule: A study with Finnish children. Int J Paediatr Dent 1993;3(4):193-8.

35. ten Berge M, Hoogstraten J, Veerkamp JS, Prins PJ. The Dental Subscale of the Children's Fear Survey Schedule: a factor analytic study in The Netherlands. Community Dent Oral Epidemiol 1998;26(5):340-3.

36. Klingberg G. Reliability and validity of the Swedish version of the Dental Subscale of the Children's
Fear Survey Schedule, CFSS-DS. Acta Odontol Scand 1994; 52(4):255-6.

37. Milgrom P, Jie Z, Yang Z, Tay KM. Cross-cultural validity of a parent's version of the Dental Fear Survey Schedule for children in Chinese. Behav Res Ther 1994;32(1):131-5.

38. Venham L, Bengston D, Cipes M. Children's response to sequential dental visits. J Dent Res 1977;56(5):454-9.

39. Buchanan H, Niven N. Validation of a Facial Image Scale to assess child dental anxiety. Int $\mathrm{J}$ Paedriatr Dent 2002;12(1):47-52.

40. Barros L, Buchanan H. Correspondence between dentist and child ratings of dental anxiety in Portugal: A preliminary study. Rev Port Estomatol Med Dent Cir Maxilofac 2011;52(1):13-5.

41. de Menezes Abreu DM, Leal SC, Mulder J, Frencken JE. Patterns of dental anxiety in children after sequential dental visits. Eur Arch Paediatr Dent 2011;12(6):298-302.

42. Sharif MO. Dental Anxiety: Detection and Management. J Appl Oral Sci 2010;18(2):i.

43. Al-Harasi S, Ashley PF, Moles DR, Parekh S, Walters V. Hypnosis for children undergoing dental treatment (Review). Cochrane Database Syst Rev 2010;4:8.

44. Bare LC, Dundes L. Strategies for Combating Dental Anxiety. J Dent Educ 2004;68(11):1172-7.

45. Rojas G, Harwardt P, Sassenfeld A, Molina Y, Herrera A, Ríos $M$ y Misrachi C. Eficacia de las técnicas de percepción de control y relajación en la reducción de ansiedad dental. Acta Odont Ven [Serie en Internet] 2011 [Citado el 13 Ago 2012];49(4); [aprox. 9 p.]. http://www.actaodontologica.com/ediciones/ 2011/4/pdf/art5.pdf.

46. Lahmann C, Schoen R, Henningsen P, Ronel J, Muehlbacher M, Loew T, Nickel M, Doering S. Brief relaxation versus music distraction in the treatment of dental anxiety: a randomized 
controlled clinical trial. J Am Dent Assoc 2008; 139(3):317-24.

47. Klassen JA, Liang Y, Tjosvold L, Klassen TP, Hartling L. Music for pain and anxiety in children undergoing medical procedures: a systematic review of randomized controlled trials. Ambul Pediatr 2008;8(2):117-28.

48. Ministerio de Salud. Guía Clínica Salud Oral Integral para Niños y Niñas de 6 años [Guía]. Santiago: MINSAL; 2008. [citado 8 de oct. 2011]. http:/ /es.scribd.com/doc/32997475/guia-clinica-saludoral-ninos-6-anos.

49. Landier W, Tse AM. Use of complementary and alternative medical interventions for the management of procedure-related pain, anxiety, and distress in pediatric oncology: an integrative review. J Pediatr Nurs 2010;25(6):566-79.

50. Wright FAC, Giebartowski JE, McMurray NE. A national survey of dentist's management of children with anxiety or behaviors problems. Austr Dent J 1991;36(5):378-83.

\section{CORRESPONDENCIA}

Matías Ríos Erazo

Sergio Livingstone P. 943, Independencia, Santiago.

Chile.

Correo electrónico: matiasrios@gmail.com 